\title{
STABLE PERTURBATION IN BANACH ALGEBRAS
}

\author{
YIFENG XUE
}

(Received 19 March 2005; revised 22 September 2006)

Communicated by A. Pryde

\begin{abstract}
Let $\mathscr{A}$ be a unital Banach algebra. Assume that $a$ has a generalized inverse $a^{+}$. Then $\bar{a}=a+\delta a \in \mathscr{A}$ is said to be a stable perturbation of $a$ if $\bar{a} \mathscr{A} \cap\left(1-a a^{+}\right) \mathscr{A}=\{0\}$. In this paper we give various conditions for stable perturbation of a generalized invertible element and show that the equation $\bar{a} \mathscr{A} \cap\left(1-a a^{+}\right) \mathscr{A}=\{0\}$ is closely related to the gap function $\hat{\delta}(\bar{a} \mathscr{A}, a \mathscr{A})$. These results will be applied to error estimates for perturbations of the Moore-Penrose inverse in $C^{*}$-algebras and the Drazin inverse in Banach algebras.
\end{abstract}

2000 Mathematics subject classification: primary 46H99, 46N40; secondary $65 \mathrm{~J} 05$.

\section{Introduction}

Throughout the paper, $\mathscr{A}$ is a complex Banach algebra with unit 1 . Let GL( $\mathscr{A})$ denote the group of all invertible elements in $\mathscr{A}$. An element $a$ in $\mathscr{A}$ is said to be generalized invertible if there is a $b \in \mathscr{A}$ such that $a b a=b$ and $b a b=b$. Such an element $b$ is called a generalized inverse of $a$, denoted by $a^{+}$(certainly such an $a^{+}$is not unique). If $\mathscr{A}$ is a $C^{*}$-algebra and $a \in \mathscr{A}$, the Moore-Penrose inverse of $a$ is defined as the element $a^{\dagger}$ satisfying

$$
a a^{\dagger} a=a, \quad a^{\dagger} a a^{\dagger}=a^{\dagger}, \quad\left(a^{\dagger} a\right)^{*}=a^{\dagger} a, \quad\left(a a^{\dagger}\right)^{*}=a a^{\dagger} .
$$

When $a \neq 0, a^{\dagger}$ is unique by [11]. We denote by $\mathrm{GI}(\mathscr{A})$ the set of all generalized invertible elements in $\mathscr{A}$. If $\mathscr{A}$ is a $C^{*}$-algebra and $a \in \mathrm{GI}(\mathscr{A})$ then, by [11, Theorem 6], $a^{\dagger}$ exists so that the set of all Moore-Penrose invertible elements in $\mathscr{A}$

Research supported by Natural Science Foundation of China and Foundation of CSC

(C) 2007 Australian Mathematical Society 1446-7887/07 \$A2.00+0.00 
is GI( $\mathscr{A})$. Recall from [9] that $a \in \mathscr{A}$ is Drazin invertible if there are a $b \in \mathscr{A}$ and a natural number $k$ such that

$$
a^{k} b a=a^{k}, \quad b a b=b, \quad a b=b a .
$$

The least $k$ such that (1.2) holds for some $b$ is called the index of $a$, denoted by Ind (a). In this case, the $b$ in (1.2) is called the Drazin inverse of $a$ and we denote it by $a^{D}$. When Ind $(a)=1, a^{D}$ is called the group inverse of $a$ and we use the symbol $a^{\#}$ to denote it; if $a \neq 0$ and Ind $(a)=0$ then $a \in \operatorname{GL}(\mathscr{A})$. Put $\mathrm{D}(\mathscr{A})=\left\{a \in \mathscr{A} \mid a^{D}\right.$ exists $\}$ and $\mathrm{G}(\mathscr{A})=\{a \in \mathrm{D}(\mathscr{A}) \mid$ Ind $(a) \leq 1\}$. It may observed that $\mathrm{GL}(\mathscr{A}) \subset \mathrm{G}(\mathscr{A}) \subset \mathrm{D}(\mathscr{A})$ and $G L(\mathscr{A}) \cup \mathrm{G}(\mathscr{A}) \subset \mathrm{GI}(\mathscr{A})$. Moreover, for any $a \in \mathrm{D}(\mathscr{A})$ and $x \in \mathrm{GL}(\mathscr{A})$,

$$
x a x^{-1} \in \mathrm{D}(\mathscr{A}), \quad \text { Ind }\left(x a x^{-1}\right)=\text { Ind }(a), \quad\left(x a x^{-1}\right)^{D}=x a^{D} x^{-1} .
$$

In recent years, many results have been published concerning the continuity of the Moore-Penrose inverse in $C^{*}$-algebras and the Drazin inverse in Banach algebras (see, for example, $[10,12,14,16])$. In $[10,16]$, Rakočević gave many equivalent conditions for the continuity of the Moore-Penrose inverse in $C^{*}$-algebras and the Drazin inverse in Banach algebras respectively. Connected with the continuity of generalized inverses and Drazin inverses, quantitative analysis of perturbations of Moore-Penrose inverses in $C^{*}$-algebras and Drazin inverses in Banach algebras has not been fully developed though Castro-González and Koliha in [2] and Rakočević and Wei in [17] have made a start on this programme.

Compared those with the study of the Moore-Penrose inverses on Hilbert spaces and Drazin inverses on Banach spaces, there are many fruitful results concerning the quantitative analysis of the perturbation of the Moore-Penrose inverses on Hilbert spaces and Drazin inverses on Banach spaces. For example, in $[5,7,20]$ the author gave an estimate of perturbation bounds for the Moore-Penrose inverse on Hilbert spaces under stable perturbation of operators, which is a generalization of rankpreserving perturbation of matrices. Meanwhile, for the Drazin inverse on Banach spaces, many perturbation analysis results have been obtained in [2], [3], [4] and [13] by means of the gap between operators (which is the gap between their graphs) or the gap between the subspaces $\operatorname{Ran}\left(T^{k}\right), \operatorname{Ran}\left(\bar{T}^{k}\right)$ and $\operatorname{Ker} T^{k}, \operatorname{Ker} \bar{T}^{k}$ of the Drazin invertible operators $T$ and $\bar{T}$ (where $k=\max \{\operatorname{Ind}(T)$, Ind $(\bar{T})\}$ ).

In order to give a quantitative analysis of perturbations of generalized inverses in $C^{*}-$ algebras and the Drazin inverse in Banach algebras without using the gap function, we first generalize the concept of the so-called stable perturbation of operators in [6] to the case of Banach algebras and establish a self-contained perturbation theory. This theory provides several useful conditions for stable perturbation. Some of these involve deep properties of idempotents in Banach algebras. Then we apply this theory 
to estimate the perturbation bounds of the Moore-Penrose inverse in $C^{*}$-algebras and the Drazin inverse in Banach algebras.

\section{Stable perturbation in Banach algebras}

Let $X$ be a Banach space and $B(X)$ be the Banach algebra of all bounded linear operators on $X$. For $T \in B(X)$, we write $\operatorname{Ran}(T)$ (respectively $\operatorname{Ker} T$ ) to denote the range (respectively null space) of $T$. Let $T \in \mathrm{GI}(B(X))$ and $\bar{T}=T+\delta T \in B(X)$. We say that $\bar{T}$ is a stable perturbation of $T$ if $\operatorname{Ran}(\bar{T}) \cap \operatorname{ker} T^{+}=\{0\}$ for some generalized inverse $T^{+}$of $T$ (cf. [6, Definition 3.1]). When $\bar{T}$ is a stable perturbation of $T$ and $\left\|T^{+}\right\|\|\delta T\|<1, \bar{T}$ has a generalized inverse of the form $\bar{T}^{+}=\left(I+T^{+} \delta T\right)^{-1} T^{+}$ (cf. [6, Corollary 3.1]). It is also noted that if $\bar{T}$ and $T$ are $m \times n$ matrices with $\left\|T^{+}\right\|\|\delta T\|<1$, then rank $\bar{T}=\operatorname{rank} T$ if and only if $\operatorname{Ran}(\bar{T}) \cap \operatorname{Ker} T^{+}=\{0\}$ (cf. $[6$, Corollary 3.2]). In short, from $[5-7,20]$ we can see that this concept plays a very important role in studying perturbations of generalized inverses in infinite-dimensional spaces.

For any $a \in \mathscr{A}$, let $L_{a}$ be the left multiplier on $\mathscr{A}$, so that $L_{a} x=a x$ for all $x \in \mathscr{A}$. Then $L_{a} \in B(\mathscr{A})$ and $\operatorname{Ran}\left(L_{a}\right)=a \mathscr{A}$ and $\operatorname{Ker} L_{a}=\{x \in \mathscr{A} \mid a x=0\}$. It is easy to check that if $a \in \mathrm{GI}(\mathscr{A})$ then $L_{a} \in \mathrm{GI}(B(\mathscr{A}))$ and $L_{a^{+}}$is one of its generalized inverses. Moreover, $\operatorname{Ker}\left(L_{a^{+}}\right)=\left(1-a a^{+}\right) \mathscr{A}$. Thus, if $\bar{a}=a+\delta a \in \mathscr{A}$ then

$$
\operatorname{Ran}\left(L_{\bar{a}}\right) \cap \operatorname{Ker}\left(L_{a^{+}}\right)=\bar{a} \mathscr{A} \cap\left(1-a a^{+}\right) \mathscr{A} .
$$

This leads to the following definition.

DEFINITION 2.1. Let $\mathscr{A}$ be a unital Banach algebra and $\bar{a}=a+\delta a \in \mathscr{A}$ for $a \in \mathrm{GI}(\mathscr{A})$. We say that $\bar{a}$ is a stable perturbation of $a$ (with respect to $a^{+}$) if $\bar{a} \mathscr{A} \cap\left(1-a a^{+}\right) \mathscr{A}=\{0\}$.

The following conditions provide means for efficient handling of stable perturbations of elements of $\mathrm{GI}(\mathscr{A})$.

PROPOSITION 2.2. Let $a \in \mathrm{GI}(\mathscr{A})$ and $\bar{a}=a+\delta a \in \mathscr{A}$ with $\left\|a^{+}\right\|\|\delta a\|<1$. Then the following conditions are equivalent.

(1) $\bar{a} \in \mathrm{GI}(\mathscr{A})$ with $\bar{a}^{+}=\left(1+a^{+} \delta a\right)^{-1} a^{+}$;

(2) $\bar{a}$ is a stable perturbation of $a$;

(3) $\bar{a}\left(1+a^{+} \delta a\right)^{-1}\left(1-a^{+} a\right)=0$;

(4) $\left(1-a a^{+}\right)\left(1+\delta a a^{+}\right)^{-1} \bar{a}=0$;

(5) $\left(1-a a^{+}\right) \delta a\left(1-a^{+} a\right)=\left(1-a a^{+}\right) \delta a\left(1+a^{+} \delta a\right)^{-1} a^{+} \delta a\left(1-a^{+} a\right)$. 
PROOF. (1) $\Rightarrow(2)$. Let $x \in \bar{a} \mathscr{A} \cap\left(1-a a^{+}\right) \mathscr{A}$. Then $a^{+} x=0$ and $x=\bar{a} y$ for some $y \in \mathscr{A}$. Put $z=\left(1+\delta a a^{+}\right) x$. Using $a^{+}\left(1+\delta a a^{+}\right)^{-1}=\left(1+a^{+} \delta a\right)^{-1} a^{+}$, we have

$$
\bar{a} \bar{a}^{+}=(a+\delta a) a^{+}\left(1+\delta a a^{+}\right)^{-1}=1-\left(1-a a^{+}\right)\left(1+\delta a a^{+}\right)^{-1} .
$$

Thus $\left(1-\bar{a} \bar{a}^{+}\right) z=x=\bar{a} y$ and hence $x=0$.

(2) $\Rightarrow(3)$. Set $z=\bar{a}\left(1+a^{+} \delta a\right)^{-1}\left(1-a^{+} a\right) \in \bar{a} \mathscr{A}$. Since

$$
a a^{+} z=a\left(1+a^{+} \delta a\right)\left(1+a^{+} \delta a\right)^{-1}\left(1-a^{+} a\right)=0,
$$

it follows that $z \in \bar{a} \mathscr{A} \cap\left(1-a a^{+}\right) \mathscr{A}=\{0\}$, so $z=0$.

$(3) \Leftrightarrow(4)$. In fact:

$$
\begin{aligned}
\left(1-a a^{+}\right)\left(1+\delta a a^{+}\right)^{-1} \bar{a} & =\left(1+\delta a a^{+}-\bar{a} a^{+}\right)\left(1+\delta a a^{+}\right)^{-1} \bar{a} \\
& =\bar{a}-\bar{a}\left(1+a^{+} \delta a\right)^{-1}\left(a^{+} \delta a+1+a^{+} a-1\right) \\
& =\bar{a}\left(1+a^{+} \delta a\right)^{-1}\left(1-a^{+} a\right) .
\end{aligned}
$$

$(3) \Rightarrow(5)$. We have

$$
\begin{aligned}
& \left(1-a a^{+}\right) \delta a\left(1+a^{+} \delta a\right)^{-1} a^{+} \delta a\left(1-a^{+} a\right) \\
& \quad=\left(1-a a^{+}\right) \delta a\left(1-a^{+} a\right)-\left(1-a a^{+}\right) \delta a\left(1+a^{+} \delta a\right)^{-1}\left(1-a^{+} a\right) \\
& \quad=\left(1-a a^{+}\right) \delta a\left(1-a^{+} a\right) .
\end{aligned}
$$

$(5) \Rightarrow(1)$. The computation above shows that if (5) is true then

$$
\left(1-a a^{+}\right) \delta a\left(1+a^{+} \delta a\right)^{-1}\left(1-a^{+} a\right)=0 .
$$

Since $a a^{+} \bar{a}\left(1+a^{+} \delta a\right)^{-1}\left(1-a^{+} a\right)=0$, we have $\bar{a}\left(1+a^{+} \delta a\right)^{-1}\left(1-a^{+} a\right)=0$. Put $b=\left(1+a^{+} \delta a\right)^{-1} a^{+}$. Then $\bar{a} b \bar{a}=\bar{a}$ and $b \bar{a} b=b$, that is, $\bar{a} \in \mathrm{GI}(\mathscr{A})$ with $\bar{a}^{+}=\left(1+a^{+} \delta a\right)^{-1} a^{+}$.

COROLLARY 2.3. Let $\mathscr{B}$ be a unital Banach subalgebra of $\mathscr{A}$. Let $a \in \mathrm{GI}(\mathscr{B})$ and $\bar{a}=a+\delta a \in \mathscr{B}$ with $\left\|a^{+}\right\|\|\delta a\|<1$. Then $\bar{a} \mathscr{B} \cap\left(1-a a^{+}\right) \mathscr{B}=\{0\}$ if and only if $\bar{a} \mathscr{A} \cap\left(1-a a^{+}\right) \mathscr{A}=\{0\}$.

PROOF. The "if" part is obvious. We now prove the "only if" part. By Proposition $2.2(1), \bar{a} \mathscr{B} \cap\left(1-a a^{+}\right) \mathscr{B}=\{0\}$ implies that $\bar{a}^{+}=\left(1+a^{+} \delta a\right)^{-1} a^{+}$for $\bar{a} \in \mathrm{GI}(\mathscr{B})$. Since $\operatorname{GI}(\mathscr{B}) \subset \mathrm{GI}(\mathscr{A})$, we have $\bar{a} \mathscr{A} \cap\left(1-a a^{+}\right) \mathscr{A}=\{0\}$ by using Proposition 2.2 again.

Let $V_{1}, V_{2}$ be subspaces in $X$. Put

$$
\delta\left(V_{1}, V_{2}\right)= \begin{cases}0, & V_{1}=\{0\} \\ \sup \left\{\operatorname{dist}\left(x, V_{2}\right) \mid x \in V_{1},\|x\|=1\right\}, & V_{1} \neq\{0\} .\end{cases}
$$

The gap function $\hat{\delta}\left(V_{1}, V_{2}\right)$ of $V_{1}$ and $V_{2}$ is defined by

$$
\hat{\delta}\left(V_{1}, V_{2}\right)=\max \left\{\delta\left(V_{1}, V_{2}\right), \delta\left(V_{2}, V_{1}\right)\right\} \text {. }
$$


By [8, Lemma 3.2], $\delta\left(V_{1}, V_{2}\right)=\delta\left(\bar{V}_{1}, \bar{V}_{2}\right)$, where $\bar{V}_{i}$ is the closure of $V_{i}$ in $X$ for $i=1,2$.

LEMMA 2.4. For idempotents $p_{1}, p_{2} \in \mathscr{A}, \hat{\delta}\left(p_{1} \mathscr{A}, p_{2} \mathscr{A}\right) \leq\left\|p_{1}-p_{2}\right\|$.

PROOF. The assertion is trivial when $p_{1}=0$. If $p_{1} \neq 0$ then for any $z \in p_{1} \mathscr{A}$ with $\|z\|=1$,

$$
\operatorname{dist}\left(z, p_{2} \mathscr{A}\right) \leq\left\|p_{1} z-p_{2} z\right\| \leq\left\|p_{1}-p_{2}\right\|
$$

Thus $\delta\left(p_{1} \mathscr{A}, p_{2} \mathscr{A}\right) \leq\left\|p_{1}-p_{2}\right\|$ and hence $\hat{\delta}\left(p_{1} \mathscr{A}, p_{2} \mathscr{A}\right) \leq\left\|p_{1}-p_{2}\right\|$.

Proposition 2.5. Let $a \in \mathrm{GI}(\mathscr{A})$ and $\bar{a}=a+\delta a \in \mathscr{A}$ with $\left\|a^{+}\right\|\|\delta a\|<1$.

(1) If $a a^{+} \neq 1$ and $\delta(\bar{a} \mathscr{A}, a \mathscr{A})<1 /\left\|1-a a^{+}\right\|$then $\bar{a} \mathscr{A} \cap\left(1-a a^{+}\right) \mathscr{A}=\{0\}$;

(2) If $\bar{a} \mathscr{A} \cap\left(1-a a^{+}\right) \mathscr{A}=\{0\}$ then

$$
\hat{\delta}(\bar{a} \mathscr{A}, a \mathscr{A}) \leq \frac{\left\|1-a a^{+}\right\|\left\|a^{+}\right\|\|\delta a\|}{1-\left\|a^{+}\right\|\|\delta a\|} .
$$

PROOF. (1) If $\bar{a} \mathscr{A} \cap\left(1-a a^{+}\right) \mathscr{A} \neq\{0\}$, we can find $x \in \bar{a} \mathscr{A} \cap\left(1-a a^{+}\right) \mathscr{A}$ with $\|x\|=1$. Then for any $y \in \mathscr{A},\left(1-a a^{+}\right)(x-a y)=x$ and it follows that $1 \leq\left\|1-a a^{+}\right\|\|x-a y\|$. Thus $\delta(\bar{a} \mathscr{A}, a \mathscr{A}) \geq\left\|1-a a^{+}\right\|^{-1}$, which contradicts the assumption.

(2) In this case, $\bar{a} \in \mathrm{GI}(\mathscr{A})$ with $\bar{a}=\left(1+a^{+} \delta a\right)^{-1} a^{+}$by Proposition 2.2 . Note that $\bar{a} \bar{a}^{+} \mathscr{A}=\bar{a} \mathscr{A}$ and $a a^{+} \mathscr{A}=a \mathscr{A}$. Therefore, by Lemma 2.4 ,

$$
\begin{aligned}
\hat{\delta}(\bar{a} \mathscr{A}, a \mathscr{A}) & \leq\left\|\bar{a} \bar{a}^{+}-a a^{+}\right\|=\left\|\left(1-a a^{+}\right)\left[1-\left(1+a^{+} \delta a\right)^{-1}\right]\right\| \\
& \leq \frac{\left\|1-a a^{+}\right\|\left\|a^{+}\right\|\|\delta a\|}{1-\left\|a^{+}\right\|\|\delta a\|} .
\end{aligned}
$$

REMARK. If $\left\|a^{+}\right\|\|\delta a\|<\left(1+\left\|1-a a^{+}\right\|^{2}\right)^{-1}$ then Proposition 2.5 shows that $\bar{a} \mathscr{A} \cap\left(1-a a^{+}\right) \mathscr{A}=\{0\}$ if and only if $\hat{\delta}(\bar{a} \mathscr{A}, a \mathscr{A})<\left\|1-a a^{+}\right\|^{-1}$.

As an application of stable perturbation in $C^{*}$-algebras, we give perturbation analysis for generalized inverses in a $C^{*}$-algebra as follows.

Proposition 2.6. Let $\mathscr{A}$ be a unital $C^{*}$-algebra. Suppose $a \in \mathrm{GI}(\mathscr{A})$ and $\bar{a}=a+\delta a \in \mathscr{A}$ with $\left\|a^{\dagger}\right\|\|\delta a\|<1$. Suppose that $\bar{a} \mathscr{A} \cap\left(1-a a^{\dagger}\right) \mathscr{A}=\{0\}$. Then $\bar{a} \in \mathrm{GI}(\mathscr{A})$ and

$$
\left\|\bar{a}^{\dagger}\right\| \leq \frac{\left\|a^{\dagger}\right\|}{1-\left\|a^{\dagger}\right\|\|\delta a\|}, \quad \frac{\left\|\bar{a}^{\dagger}-a^{\dagger}\right\|}{\left\|a^{\dagger}\right\|} \leq \frac{1+\sqrt{5}}{2} \frac{\left\|a^{\dagger}\right\|\|\delta a\|}{1-\left\|a^{\dagger}\right\|\|\delta a\|} .
$$


PROOF. By [15], $\mathscr{A}$ has a faithful representation $\left(\pi, H_{\pi}\right)$ such that $\pi(\mathscr{A})$ is a unital $C^{*}$-subalgebra of $B(H)$ with $I=\pi(1)$. Put $A=\pi(a), \bar{A}=\pi(\bar{a}), \delta A=\bar{A}-A$. Then $\bar{A}\left[B\left(H_{\pi}\right)\right] \cap\left(I-A A^{\dagger}\right)\left[B\left(H_{\pi}\right)\right]=\{0\}$ by Corollary 2.3. Let $\eta \in \operatorname{Ran}(\bar{A}) \cap \operatorname{Ker} A^{\dagger}$. Then $A^{\dagger} \eta=0$ and $\eta=\bar{A} \xi_{0}$ for some $\xi_{0} \in H_{\pi}$. Choose a nonzero vector $\xi_{1} \in H_{\pi}$ and put $A_{1} \xi=\left(\xi, \xi_{1}\right) \xi_{0}, A_{2} \xi=\left(\xi, \xi_{1}\right) \eta$ for all $\xi \in H_{\pi}$. Then $A_{2}=\bar{A} A_{1}$ and $A_{2}=\left(I-A A^{\dagger}\right) A_{2}$. Thus

$$
A_{2} \in \bar{A}\left[B\left(H_{\pi}\right)\right] \cap\left(I-A A^{\dagger}\right)\left[B\left(H_{\pi}\right)\right]=\{0\}
$$

so that $\eta=0$, that is, $\vec{A}$ is a stable perturbation of $A$ as operators on $H_{\pi}$. Therefore $\bar{A}^{\dagger}$ exists and $\left\|\bar{A}^{\dagger}\right\| \leq\left\|A^{\dagger}\right\| /\left(1-\left\|A^{\dagger}\right\|\|\delta A\|\right)$ by $[7$, Theorem 1] and

$$
\frac{\left\|\bar{A}^{\dagger}-A^{\dagger}\right\|}{\left\|A^{\dagger}\right\|} \leq \frac{1+\sqrt{5}}{2} \frac{\left\|A^{\dagger}\right\|\|\delta A\|}{1-\left\|A^{\dagger}\right\|\|\delta A\|}
$$

by [20, Proposition 7].

Since $\pi$ is a $*$-isometry and 0 is an isolated point of the spectrum of $\bar{A}^{*} \bar{A}$, it follows that 0 is an isolated point of the spectrum of $\bar{a}^{*} \bar{a}$, that is, $\bar{a} \in \mathrm{GI}(\mathscr{A})$ and (2.1) follows.

Let $\mathscr{A}$ be a unital $C^{*-}$-algebra and $\left\{a_{n}\right\}_{0}^{\infty} \subset \mathrm{GI}(\mathscr{A}) \backslash\{0\}$ with $\lim _{n \rightarrow \infty} a_{n}=a_{0}$. In [16], Rakočević gave various equivalent conditions that make $\lim _{n \rightarrow \infty} a_{n}^{\dagger}=a_{0}^{\dagger}$ in $\mathscr{A}$. Combining [16, Theorem 2.2] with Lemma 2.4 and Proposition 2.6, we have the following result.

COROLlaRY 2.7. Let $\mathscr{A}$ be a unital $C^{*}$-algebra and $\left\{a_{n}\right\}_{0}^{\infty} \subset \mathrm{GI}(\mathscr{A}) \backslash\{0\}$ with $\lim _{n \rightarrow \infty} a_{n}=a_{0}$. Then following conditions are equivalent:

(1) $a_{n}^{\dagger} \rightarrow a_{0}$;

(2) $a_{n} a_{n}^{\dagger} \rightarrow a_{0} a_{0}^{\dagger}$;

(3) $a_{n}^{\dagger} a_{n} \rightarrow a_{0}^{\dagger} a_{0}$;

(4) $\sup _{n}\left\|a_{n}^{\dagger}\right\|<+\infty$;

(5) $a_{n} \mathscr{A} \cap\left(1-a_{0} a_{0}^{\dagger}\right) \mathscr{A}=\{0\}$ for $n$ large enough.

PROOF. The proof of the equivalence of (1), (2), (3) and (4) follows from [16, Theorem 2.2].

(3) $\Rightarrow(5)$. By Lemma 2.4,

$$
\hat{\delta}\left(a_{n} \mathscr{A}, a_{0} \mathscr{A}\right)=\hat{\delta}\left(a_{n} a_{n}^{\dagger} \mathscr{A}, a_{0} a_{0}^{\dagger} \mathscr{A}\right) \leq\left\|a_{n} a_{n}^{\dagger}-a_{0} a_{0}^{\dagger}\right\| .
$$

So $\lim _{n \rightarrow \infty} \hat{\delta}\left(a_{n} \mathscr{A}, a_{0} \mathscr{A}\right)=0$ and hence, $a_{n} \mathscr{A} \cap\left(1-a_{0} a_{0}^{\dagger}\right) \mathscr{A}=\{0\}$ for $n$ large enough by Proposition 2.5 (take $\delta a_{0}=a_{n}-a_{0}$ and $\bar{a}_{0}=a_{0}+\delta a_{0}$ ).

(5) $\Rightarrow(1)$. When $a_{n} \mathscr{A} \cap\left(1-a_{0} a_{0}^{\dagger}\right) \mathscr{A}=\{0\}$ for sufficiently large $n$, we have $\lim _{n \rightarrow \infty} a_{n}^{\dagger}=a_{0}^{\dagger}$ by Proposition 2.6. 


\section{Some further conditions for stable perturbation}

We know from [6, Corollary 3.1] that, for $T, \bar{T}=T+\delta T \in B(X)$ with $T^{+}$existing and $\left\|T^{+}\right\|\|\delta T\|<1$, if $\operatorname{dim} \operatorname{Ker} \bar{T}=\operatorname{dim} \operatorname{Ker} T<+\infty$ (or $\operatorname{rank} \bar{T}=\operatorname{rank} T$ when $\operatorname{dim} X<+\infty)$ then $\bar{T}$ is a stable perturbation of $T$. We will extend this result to the case of Banach algebras.

For $a \in \mathscr{A}$, put $\operatorname{Ker}(a)=\{x \in \mathscr{A} \mid a x=0\}$ and $\operatorname{coKer}(a)=\{x \in \mathscr{A} \mid x a=0\}$. Obviously, if $a \in \mathrm{GI}(\mathscr{A})$ then $\operatorname{Ker}(a)=\left(1-a^{+} a\right) \mathscr{A}$ and $\operatorname{coKer}(a)=\mathscr{A}\left(1-a a^{+}\right)$.

LEMMA 3.1. Let $a \in \mathrm{GI}(\mathscr{A})$ and $\bar{a}=a+\delta a \in \mathscr{A}$ with $\left\|a^{+}\right\|\|\delta a\|<1$. Put

$$
p_{a}=\left(1+a^{+} \delta a\right)^{-1}\left(1-a^{+} a\right), \quad q_{a}=\left(1-a a^{+}\right)\left(1+\delta a a^{+}\right)^{-1} .
$$

Then $\operatorname{Ker}(\bar{a}) \subset p_{a} \mathscr{A}$ and $\operatorname{coKer}(\bar{a}) \subset \mathscr{A} q_{a}$.

PROOF. Let $x \in \operatorname{Ker}(\bar{a})$ and $y \in \operatorname{coKer}(\bar{a})$. Then

$$
\left(a^{+} a-1+1+a^{+} \delta a\right) x=0, \quad y\left(a a^{+}-1+1+\delta a a^{+}\right)=0 .
$$

Consequently, $x=p_{a} x, y=y q_{a}$, that is, $\operatorname{Ker}(\bar{a}) \subset p_{a} \mathscr{A}$ and $\operatorname{coKer}(\bar{a}) \subset \mathscr{A} q_{a}$.

Let $p, q$ be idempotents in a complex Banach algebra $\mathscr{E}$. Recall that $p$ and $q$ are equivalent in $\mathscr{E}$ (in symbols, $p \sim q$ ) if there are $x, y \in \mathscr{E}$ such that $p=x y, q=y x$. Note that $x, y$ can be chosen so that $p x=x q=x$ and $q y=y p=y$. Also $p$ and $q$ are said to be similar (in symbols, $p \approx q$ ), if there is an $a \in \mathrm{GL}(\mathscr{E})$ such that $p=a^{-1} q a$ when $\mathscr{E}$ is unital; if $\mathscr{E}$ is nonunital $p \approx q$ means that there is an $a \in \operatorname{GL}(\widetilde{\mathscr{E}})$ such that $p=a^{-1} q a$, where $\widetilde{\mathscr{E}}=\{\lambda+x \mid \lambda \in \mathbb{C}, x \in \mathscr{E}\}$.

Clearly, if $p \approx q$, then $p \sim q$.

Let $\mathscr{E}$ be a Banach algebra with unit 1 . $\mathscr{E}$ is said to be finite if for any idempotent $e \in \mathscr{E}$ with $e \sim 1$, we have $e=1$. If $\mathscr{E}$ is nonunital and $\widetilde{E}$ is finite, we will say $\mathscr{E}$ is finite. For example, every finite dimensional Banach algebra is finite; the matrix algebra over a commutative Banach algebra is finite; and the Banach algebra $\mathscr{K}(X)$ consisting of all compact operators on $X$ is finite (this can be shown by means of the Fredholm index).

Let $p$ be a nonzero idempotent in $\mathscr{E}$. We will say $p$ is finite if $p \mathscr{E} p$ is finite.

LEMMA 3.2. Let $p, q, r$ be nonzero idempotents in a Banach algebra $\mathscr{E}$.

(1) If $p \sim q$ and $q \sim r$ then $p \sim r$;

(2) If $p$ is finite and $p q=q p=q$ and $p \sim q$ then $p=q$;

(3) If $p$ is finite and $p \sim q$ then $q$ is finite. 
ProOF. (1) Let $x_{1}, y_{1}, x_{2}, y_{2} \in \mathscr{E}$ be such that

$$
p=x_{1} y_{1}, \quad q=y_{1} x_{1}, \quad q=x_{2} y_{2}, \quad r=y_{2} x_{2} .
$$

Put $z_{1}=x_{1} x_{2}, z_{2}=y_{2} y_{1}$. Then $z_{1} z_{2}=p, z_{2} z_{1}=r$.

(2) Let $x, y \in \mathscr{E}$ be such that

$$
p=x y, \quad q=y x, \quad p x=x q=x, \quad q y=y p=y .
$$

Since $p q=q p=q$, it follows that $x=p x p, y=p y p$. Thus $p \sim q$ in $p \mathscr{E} p$. Noting that $p$ is the unit of $p \mathscr{E} p$ and $p \mathscr{E} p$ is finite, we have $p=q$.

(3) Let $e$ be an idempotent in $q \mathscr{E} q$ with $e \sim q$. Let $a, b \in \mathscr{E}$ be such that

$$
p=a b, \quad q=b a, \quad p a=a q=a, \quad q b=b p=b .
$$

Put $f=a e b$. Then $f p=p f=f$ by (3.1). From $e q=q e=e$, we get that $f^{2}=f$. Since $f \sim e \sim q \sim p$ by (1) and $p$ is finite, we have $f=p$ by (2) and hence $e=q$ by (3.1).

THEOREM 3.3. Let $\mathscr{A}$ be a unital Banach algebra and let $a, \bar{a}=a+\delta a \in \mathrm{GI}(\mathscr{A})$ with $\left\|a^{+}\right\|\|\delta a\|<1$. If $1-a^{+} a$ (or $\left.1-a a^{+}\right)$is finite and $1-a^{+} a \sim 1-\bar{a}^{+} \bar{a}$ (or $\left.1-a a^{+} \sim 1-\bar{a} \bar{a}^{+}\right)$, then $\bar{a} \mathscr{A} \cap\left(1-a a^{+}\right) \mathscr{A}=\{0\}$.

PROOF. Let $p_{a}, q_{a}$ be as in Lemma 3.1. From $p_{a}=\left(1+a^{+} \delta a\right)^{-1}\left(1-a^{+} a\right)\left(1+a^{+} \delta a\right), \quad q_{a}=\left(1+\delta a a^{+}\right)\left(1-a a^{+}\right)\left(1+\delta a a^{+}\right)^{-1}$, we see that $p_{a}$ and $q_{a}$ are idempotents and $p_{a} \approx 1-a^{+} a, q_{a} \approx 1-a a^{+}$.

Now put $\bar{p}=1-\bar{a}^{+} \bar{a}, \bar{q}=1-\bar{a} \bar{a}^{+}$. Then, by Lemma 3.1, $p_{a} \bar{p}=\bar{p}$ and $\bar{q} q_{a}=\bar{q}$. Set $w_{1}=1+\bar{p} p_{a}(1-\bar{p}), w_{2}=1+(1-\bar{q}) q_{a} \bar{q}$. Then $w_{1}, w_{2} \in \operatorname{GL}(\mathscr{A})$ with $w_{1}^{-1}=1-\bar{p} p_{a}(1-\bar{p}), w_{2}^{-1}=1-(1-\bar{q}) q_{a} \bar{q}$. Set $\bar{p}_{1}=w_{1} p_{a} w_{1}^{-1}, \bar{q}_{1}=w_{2}^{-1} q_{a} w_{2}$. Using $p_{a} \bar{p}=\bar{p}, \bar{q} q_{a}=\bar{q}$, we obtain

$$
\begin{array}{ll}
\bar{p}_{1}=p_{a}-\bar{p} p_{a}(1-\bar{p}), & \bar{p}_{1} \bar{p}=\bar{p} \bar{p}_{1}=\bar{p} \\
\bar{q}_{1}=q_{a}-(1-\bar{q}) q_{a} \bar{q}, & \bar{q}_{1} \bar{q}=\bar{q} \bar{q}_{1}=\bar{q} .
\end{array}
$$

Since $1-a^{+} a \sim \bar{p}_{1} \sim \bar{p}$ (or $1-a a^{+} \sim \bar{q}_{1} \sim \bar{q}$ ) and $1-a^{+} a$ (or $1-a a^{+}$) is finite, it follows from (3.1) (or (3.2)) and Lemma 3.2 that $\bar{p}_{1}=\bar{p}$ (or $\bar{q}_{1}=\bar{q}$ ), that is, $p=\bar{p} p$ (or $q=q \bar{q}$ ) and hence

$$
\bar{a}\left(1+a^{+} \delta a\right)^{-1}\left(1-a^{+} a\right)=0 \quad\left(\text { or }\left(1-a a^{+}\right)\left(1+\delta a a^{+}\right)^{-1} \bar{a}=0\right) .
$$

Therefore $\bar{a} \mathscr{A} \cap\left(1-a a^{+}\right) \mathscr{A}=\{0\}$ by Proposition 2.2 . 
Let $p$ be a nonzero idempotent in Banach algebra $\mathscr{E}$. We say $p$ is minimal if $p \mathscr{E} p=\{\lambda p \mid \lambda \in \mathbb{C}\}$. Let $M(\mathscr{E})$ denote the set of all minimal idempotents in $\mathscr{E}$. Now suppose that $\mathscr{E}$ is a semiprime Banach algebra (that is, for any $x \in \mathscr{E} \backslash\{0\}$ there is $y \in \mathscr{E}$ such that $y x y \neq 0$ ) and $M(\mathscr{E}) \neq \emptyset$. Then the socle $S_{\mathscr{E}}$ is defined as the smallest ideal which contains $\{\mathscr{E} p \mid p \in M(\mathscr{E})\}$ (or $\{p \mathscr{E} \mid p \in M(\mathscr{E})\}$ ) (cf. [18, pages 45-47]).

According to [1], a right (or left) ideal $M$ of $\mathscr{E}$ is of finite order if $M$ is the sum of a finite number of minimal right (or left) ideals of $\mathscr{E}$. The order $\theta(M)$ of $M$ is defined as the smallest number of minimal right (or left) ideals which have sum $M$ when $M \neq\{0\}$ and $\theta(M)=0$ if $M=\{0\}$. By [1, Lemma 1.1], $\theta(M)=m \geq 1$ if and only if there are $e_{1}, \cdots, e_{m} \in M(\mathscr{E}) \cap M$ with $e_{i} e_{j}=0, i \neq j, i, j=1, \cdots, m$ such that $M=\left(\sum_{i=1}^{m} e_{i}\right) \mathscr{E}\left(\right.$ or $\left.\mathscr{E}\left(\sum_{i=1}^{m} e_{i}\right)\right)$.

THEOREM 3.4. Let $\mathscr{A}$ be a unital Banach algebra with $M(\mathscr{A}) \neq \emptyset$. Let $a \in \mathrm{GI}(\mathscr{A})$ and $\vec{a}=a+\delta a \in \mathscr{A}$ with $\left\|a^{+}\right\|\|\delta a\|<1$. Assume that $\operatorname{Ker}(a)$ (or $\left.\operatorname{coKer}(a)\right)$ is of finite order. Then so is the $\operatorname{Ker}(\bar{a})$ (or coKer $(\bar{a}))$.

In addition, if $\theta(\operatorname{Ker}(\bar{a}))=\theta(\operatorname{Ker}(a))(\operatorname{or} \theta(\operatorname{coKer}(\bar{a}))=\theta(\operatorname{coKer}(a)))$ then $\bar{a}$ is stable perturbation of $a$.

PROOF. We only give the proof for right ideals $\operatorname{Ker}(a)$ and $\operatorname{Ker}(\bar{a})$. The proof of the remainder is similar.

Let $p_{a}=\left(1+a^{+} \delta a\right)^{-1}\left(1-a^{+} a\right)$. Since $p_{a} \approx 1-a^{+} a$, we have

$$
\theta\left(p_{a} \mathscr{A}\right)=\theta\left(\left(1-a^{+} a\right) \mathscr{A}\right)=\theta(\operatorname{Ker}(a))<+\infty .
$$

Suppose that $\theta(\operatorname{Ker}(a))=n \geq 1$. Then there are $e_{1}, \ldots, e_{n} \in M(\mathscr{A}) \cap p_{a} \mathscr{A}$ such that

$$
p_{a} \mathscr{A}=e_{1} \mathscr{A}+\cdots+e_{n} \mathscr{A}, \quad e_{i} e_{j}=0, i \neq j, i, j=1, \ldots, n .
$$

Put $e=\sum_{i=1}^{n} e_{i}$. Then $p_{a} \mathscr{A}=e \mathscr{A}$, that is, $p_{a} e=e, e p_{a}=p_{a}$. Since $\operatorname{Ker}(\bar{a}) \subset p_{a} \mathscr{A}$ by Lemma 3.1, we have

$$
e[\operatorname{Ker}(\bar{a})]=\operatorname{Ker}(\bar{a}) \quad \text { and by (3.4) } \quad e_{i}[\operatorname{Ker}(\bar{a})] \subset e_{i} \mathscr{A}, \quad i=1, \ldots, n .
$$

Assume that $\operatorname{Ker}(\bar{a}) \neq\{0\}$. Since both $e_{i}[\operatorname{Ker}(\bar{a})]$ and $e_{i} \mathscr{A}$ are right ideals and $e_{i} \mathscr{A}$ is minimal, it follows that

$$
e_{i}[\operatorname{Ker}(\bar{a})]=\{0\} \quad \text { or } \quad e_{i}[\operatorname{Ker}(\bar{a})]=e_{i} \mathscr{A}, \quad i=1, \ldots, n .
$$

Without loss of generality, we may assume that $e_{i}[\operatorname{Ker}(\bar{a})]=e_{i} \mathscr{A}$ for $i=1, \ldots, k$ and $e_{i}[\operatorname{Ker}(\bar{a})]=\{0\}$ for $i=k+1, \ldots, n$. Put $e^{\prime}=\sum_{i=1}^{k} e_{i}$. Then

$$
\operatorname{Ker}(\bar{a})=e[\operatorname{Ker}(\bar{a})]=e^{\prime}[\operatorname{Ker}(\bar{a})]=e^{\prime} \mathscr{A} .
$$


This means that $\theta(\operatorname{Ker}(\bar{a}))=k \leq n$.

If $\theta(\operatorname{Ker}(\bar{a}))=\theta(\operatorname{Ker}(a))$, we have $\operatorname{Ker}(\bar{a})=e \mathscr{A}=p_{a} \mathscr{A}$ by the above argument. Thus $\bar{a}\left(1+a^{+} \delta a\right)^{-1}\left(1-a^{+} a\right)=0$ and hence $\bar{a} \mathscr{A} \cap\left(1-a a^{+}\right) \mathscr{A}=\{0\}$ by Proposition 2.2.

\section{Perturbation analysis for Drazin inverse}

Let $a \in G(\mathscr{A})$ and put $a^{\pi}=1-a a^{\#}$. Then $a^{\pi}$ is an idempotent and $a a^{\pi}=a^{\pi} a=0$ and $a^{\#} a^{\pi}=a^{\pi} a^{\#}=0$. Let $\bar{a}=a+\delta a \in \mathscr{A}$ with $\kappa_{\#}(a) \epsilon_{a}<\left(1+\left\|a^{\pi}\right\|\right)^{-1}$, where $\kappa_{\#}(a)=\|a\|\left\|a^{\#}\right\|$ and $\epsilon_{a}=\|\delta a\|\|a\|^{-1}$. Put

Since

$$
\Phi(a)=1+\delta a\left(1-a a^{\#}\right) \delta a\left[\left(I+a^{\#} \delta a\right)^{-1} a^{\#}\right]^{2} .
$$

$$
\|1-\Phi(a)\| \leq\left\|a^{\pi}\right\|\left(\frac{\kappa_{\#}(a) \epsilon_{a}}{1-\kappa_{\#}(a) \epsilon_{a}}\right)^{2}<1,
$$

it follows that $\Phi(a) \in \mathrm{GL}(\mathscr{A})$ and $\Phi^{-1}(a)=(\Phi(a))^{-1}=\sum_{n=0}^{\infty}(1-\Phi(a))^{n}$ with

$$
\left\|\Phi^{-1}(a)\right\| \leq \frac{\left(1-\kappa_{\sharp}(a) \epsilon_{a}\right)^{2}}{\left(1-\kappa_{\sharp}(a) \epsilon_{a}\right)^{2}-\left\|a^{\pi}\right\|\left(\kappa_{\#}(a) \epsilon_{a}\right)^{2}} .
$$

LEMMA 4.1. Let $a \in G(\mathscr{A})$ and $\bar{a}=a+\delta a \in \mathscr{A}$ with $\kappa_{\#}(a) \epsilon_{a}<\left(1+\left\|a^{\pi}\right\|\right)^{-1}$. Put

$$
C(a)=a^{\pi} \delta a\left(1+a^{\#} \delta a\right)^{-1} a^{\#}, \quad D(a)=\left(1+a^{\#} \delta a\right)^{-1} a^{\#} \Phi^{-1}(a) .
$$

If $\bar{a} \cap\left(1-a a^{\#}\right) \mathscr{A}=\{0\}$ then $\bar{a} \in G(\mathscr{A})$ with

$$
\bar{a}^{\#}=(1+C(a))\left(D(a)+D^{2}(a) \delta a a^{\pi}\right)(1-C(a)) .
$$

Proof. Clearly, $1+C(a) \in \mathrm{GL}(\mathscr{A})$ with $(1+C(a))^{-1}=1-C(a)$. Applying conditions (3) and (5) from Proposition 2.2 to $\bar{a}_{0}=(1-C(a)) \bar{a}(1+C(a))$, we have

$$
\begin{aligned}
\bar{a}_{0} & =a+a a^{\#} \delta a a a^{\#}+a a^{\#} \delta a a^{\pi} \delta a\left(1+a^{\#} \delta a\right) a^{\#}+a a^{\#} \delta a a^{\pi} \\
& =a a^{\#} \Phi(a) a\left(1+a^{\#} \delta a\right) a a^{\#}+a a^{\#} \delta a a^{\pi} .
\end{aligned}
$$

Noting that $a^{\pi}\left(1+a^{\#} \delta a\right)^{-1} a^{\#}=0$ and $\Phi(a) a^{\pi}=a^{\pi}$, it can be checked that

$$
\begin{aligned}
\bar{a}_{0}^{\#} & =\left(1+a^{\#} \delta a\right)^{-1} a^{\#} \Phi^{-1}(a)+\left[\left(1+a^{\#} \delta a\right)^{-1} a^{\#} \Phi^{-1}(a)\right]^{2} \delta a a^{\pi} \\
& =D(a)+D^{2}(a) \delta a a^{\pi} .
\end{aligned}
$$

Therefore $\bar{a}^{\#}=(1+C(a))\left(D(a)+D^{2}(a) \delta a a^{\pi}\right)(1-C(a))$. 
We now give a perturbation analysis for group inverses under stable perturbation as follows.

THEOREM 4.2. Let $a \in G(\mathscr{A})$ and $\bar{a}=a+\delta a \in \mathscr{A}$ with $\kappa_{\sharp}(a) \epsilon_{a}<\left(1+\left\|a^{\pi}\right\|\right)^{-1}$. Assume that $\bar{a} \mathscr{A} \cap\left(1-a a^{\#}\right) \mathscr{A}=\{0\}$. Then $\bar{a} \in G(\mathscr{A})$ and

$$
\left\|\bar{a}^{\#}\right\| \leq \frac{\left\|a^{\#}\right\|}{\left[1-\left(1+\left\|a^{\pi}\right\|\right) \kappa_{\#}(a) \epsilon_{a}\right]^{2}}, \quad \frac{\left\|\bar{a}^{\#}-a^{\#}\right\|}{\left\|a^{\#}\right\|} \leq \frac{\left(1+2\left\|a^{\pi}\right\|\right) \kappa_{\sharp}(a) \epsilon_{a}}{\left[1-\left(1+\left\|a^{\pi}\right\|\right) \kappa_{\#}(a) \epsilon_{a}\right]^{2}} .
$$

Proof. We keep $C(a), D(a)$ as in Lemma 4.1. Then, by Lemma 4.1, $\bar{a} \in G(\mathscr{A})$. Since $D(a)(I-C(a))=D(a)$, it follows from (4.2) that

$$
\begin{aligned}
\bar{a}^{\#} & =(I+C(a)) D(a)+(I+C(a)) D^{2}(a) \delta a a^{\pi}(I-C(a)), \\
\left\|\bar{a}^{\#}\right\| & \leq(1+\|C(a)\|)\|D(a)\|+(1+\|C(a)\|)^{2}\|D(a)\|^{2}\|\delta a\|\left\|a^{\pi}\right\| .
\end{aligned}
$$

We have, by (4.1),

Since

$$
\|D(a)\| \leq \frac{\left(1-\kappa_{\#}(a) \epsilon_{a}\right)\left\|a^{\#}\right\|}{\left[1-\left(1+\left\|a^{\pi}\right\|\right) \kappa_{\#}(a) \epsilon_{a}\right]\left[1+\left(\left\|a^{\pi}\right\|-1\right) \kappa_{\#}(a) \epsilon_{a}\right]} .
$$

we deduce that

$$
1+\|C(a)\| \leq \frac{1+\left(\left\|a^{\pi}\right\|-1\right) \kappa_{\#}(a) \epsilon_{a}}{1-\kappa_{\sharp}(a) \epsilon_{a}}
$$

$$
\left\|\bar{a}^{\#}\right\| \leq \frac{\left(1-\kappa_{\#}(a) \epsilon_{a}\right)\left\|a^{\#}\right\|}{\left[1-\left(1+\left\|a^{\pi}\right\|\right) \kappa_{\#}(a) \epsilon_{a}\right]^{2}} \leq \frac{\left\|a^{\#}\right\|}{\left[1-\left(1+\left\|a^{\pi}\right\|\right) \kappa_{\#}(a) \epsilon_{a}\right]^{2}}
$$

by (4.4). Finally, by (4.3),

$$
\left\|\bar{a}^{\#}-a^{\#}\right\| \leq\left\|D(a)-a^{\#}\right\|+\|C(a) D(a)\|+(1+\|C(a)\|)^{2}\|D(a)\|^{2}\|\delta a\|\left\|a^{\pi}\right\| .
$$

Now we have

$$
\begin{aligned}
\left\|D(a)-a^{\#}\right\| & =\left\|\left[\left(1+a^{\pi} \delta a\right)^{-1} a^{\#}-a^{\#}\right] \Phi^{-1}(a)+a^{\#}\left(\Phi^{-1}(a)-1\right)\right\| \\
& \leq \frac{\left\|a^{\#}\right\| \kappa_{\#}(a) \epsilon_{a}}{1-\kappa_{\#}(a) \epsilon_{a}}\left\|\Phi^{-1}(a)\right\|+\left\|a^{\#}\right\|\left\|\Phi^{-1}(a)\right\|\|\Phi(a)-1\| \\
& \leq \frac{\left\|a^{\#}\right\| \kappa_{\#}(a) \epsilon_{a}}{1-\left(1+\left\|a^{\pi}\right\|\right) \kappa_{\#}(a) \epsilon_{a}}, \\
\|C(a) D(a)\| & \leq \frac{\left\|a^{\pi}\right\| \kappa_{\#}(a) \epsilon_{a}}{1-\kappa_{\#}(a) \epsilon_{a}} \frac{\left(1-\kappa_{\#}(a) \epsilon_{a}\right)\left\|a^{\#}\right\|}{1-2 \kappa_{\#}(a) \epsilon_{a}-\left(\left\|a^{\pi}\right\|-1\right)\left(\kappa_{\#}(a) \epsilon_{a}\right)^{2}} \\
& \leq \frac{\left\|a^{\sharp}\right\|\left\|a^{\pi}\right\| \kappa_{\#}(a) \epsilon_{a}}{1-\left(1+\left\|a^{\pi}\right\|\right) \kappa_{\#}(a) \epsilon_{a}}\left(\left(\kappa_{\#}(a) \epsilon_{a}\right)^{2} \leq \kappa_{\sharp}(a) \epsilon_{a}<1\right) .
\end{aligned}
$$

Therefore

$$
\left\|\bar{a}^{\#}-a^{\#}\right\| \leq \frac{\left(1+2\left\|a^{\pi}\right\|\right)\left\|a^{\#}\right\| \kappa_{\#}(a) \epsilon_{a}}{\left[1-\left(1+\left\|a^{\pi}\right\|\right) \kappa_{\#}(a) \epsilon_{a}\right]^{2}} .
$$


Let $a, \bar{a}=a+\delta a \in \mathscr{A}$ and put $\delta a^{j}=(a+\delta a)^{j}-a^{j}, j=1, \ldots, n$. Then

$$
\left\|\delta a^{j}\right\| \leq(\|a\|+\|\delta a\|)^{j-1}\|\delta a\|+\|a\|\left\|\delta a^{j-1}\right\| .
$$

Suppose $\epsilon_{a}<1$. From above, we can deduce that

$$
\left\|\delta a^{n}\right\| \leq\|\delta a\| \sum_{j=0}^{n-1}\|a\|^{n-1}\left(1+\epsilon_{a}\right)^{n-1-j}<\left(2^{n}-1\right)\|a\|^{n} \epsilon_{a} .
$$

Let $a \in D(\mathscr{A})$ with Ind $(a)=k$. Put $a^{\pi}=1-a a^{D}, \kappa_{D}(a)=\|a\|\left\|a^{D}\right\|$. It is well-known that $\left(a^{n}\right)^{\#}=\left(a^{D}\right)^{n}, a^{n}\left(a^{D}\right)^{n}=1-a^{\pi}$ and $a^{D}=\left(a^{n}\right)^{\#} a^{n-1}$ for any $n \geq k$. So $\operatorname{Ker}\left(a^{n}\right)=a^{\pi} \mathscr{A}$ and $a^{n} \mathscr{A}=\left(1-a^{\pi}\right) \mathscr{A}$ for all $n \geq k$.

Using Theorem 4.2, we can give perturbation bounds for Drazin invertible elements in a Banach algebra $\mathscr{A}$ as follows.

COROLlARY 4.3. Let $a, \bar{a}=a+\delta a \in D(\mathscr{A})$ with Ind $(a)=k_{1}$, Ind $(\bar{a})=k_{2}$ and

$$
\kappa_{D}^{n}(a) \epsilon_{a}<\frac{1}{\left(2^{n}-1\right)\left(1+\left\|a^{\pi}\right\|\right)}, \quad \text { where } n=\max \left\{k_{1}, k_{2}\right\} .
$$

If $\left(1-\bar{a}^{\pi}\right) \mathscr{A} \cap a^{\pi} \mathscr{A}=\{0\}$ then

$$
\begin{aligned}
\left\|\bar{a}^{D}\right\| & \leq \frac{2^{n-1} \kappa_{D}^{n-1}(a)\left\|a^{D}\right\|}{\left[1-\left(2^{n}-1\right)\left(1+\left\|a^{\pi}\right\|\right) \kappa_{D}^{n}(a) \epsilon_{a}\right]^{2}}, \\
\frac{\left\|\bar{a}^{D}-a^{D}\right\|}{\left\|a^{D}\right\|} & \leq \frac{2^{n-1}\left(2^{n}-1\right)\left(1+2\left\|a^{\pi}\right\|\right) \kappa_{D}^{2 n-1}(a) \epsilon_{a}}{\left[1-\left(2^{n}-1\right)\left(1+\left\|a^{\pi}\right\|\right) \kappa_{D}^{n}(a) \epsilon_{a}\right]^{2}}+\left(2^{n-1}-1\right) \kappa_{D}^{n-1}(a) \epsilon_{a} .
\end{aligned}
$$

PROOF. Noting that $\kappa_{D}(a) \geq\left\|a a^{D}\right\| \geq 1$, we have $\epsilon_{a}<1$ and

$$
\left\|\delta a^{n}\right\|\left\|\left(a^{n}\right)^{\#}\right\|<\left(2^{n}-1\right) \kappa_{D}^{n}(a) \epsilon_{a}<\frac{1}{1+\left\|a^{\pi}\right\|}
$$

by (4.6). Since $\vec{a}^{D}=\left(\bar{a}^{n}\right)^{\#} \bar{a}^{n-1}, a^{D}=\left(a^{n}\right)^{\#} a^{n-1}$, it follows that

$$
\begin{aligned}
\left\|\bar{a}^{D}\right\| & \leq\left\|\left(\bar{a}^{n}\right)^{\#}\right\|\|a\|^{n-1}\left(1+\epsilon_{a}\right)^{n-1}<2^{n-1}\|a\|^{n-1}\left\|\left(\bar{a}^{n}\right)^{\#}\right\|, \\
\left\|\bar{a}^{D}-a^{D}\right\| & \leq\left\|\left(\bar{a}^{n}\right)^{\#}-\left(a^{n}\right)^{\#}\right\|\|\bar{a}\|^{n-1}+\left\|\left(a^{n}\right)^{\#}\right\|\left\|\delta a^{n-1}\right\| \\
& <2^{n-1}\left\|\left(\bar{a}^{n}\right)^{\#}-\left(a^{n}\right)^{\#}\right\|\|a\|^{n-1}+\left(2^{n-1}-1\right)\left\|a^{D}\right\| \kappa_{D}^{n-1}(a) \epsilon_{a}
\end{aligned}
$$

by (4.6). Then applying Theorem 4.2 to $\left(a^{n}\right)^{\#}$ and $\left(\bar{a}^{n}\right)^{\#}$, we obtain the assertion.

Let $\left\{a_{n}\right\}_{n=0}^{\infty} \subset D(\mathscr{A})$ and $\lim _{n \rightarrow \infty} a_{n}=a_{0}$. By [10, Theorem 4.1], $\lim _{n \rightarrow \infty} a_{n}^{D}=a_{0}^{D}$ if and only if $\lim _{n \rightarrow \infty} a_{n}^{D} a_{n}=a_{0}^{D} a_{0}$, if and only if $\sup _{n \geq 1}\left\|a_{n}^{D}\right\|<+\infty$, etc. In addition, if $\sup _{n \geq 0}$ Ind $\left(a_{n}\right)<+\infty$, we have the following. 
COROLlaRY 4.4. Let $a_{n} \in D(\mathscr{A})$ for $n \geq 0$ with $\lim _{n \rightarrow \infty} a_{n}=a_{0}$ and suppose that $l=\sup _{n \geq 0}$ Ind $\left(a_{n}\right)<+\infty$.

(1) $\lim _{n \rightarrow \infty} a_{n}^{D}=a_{0}^{D}$ if and only if $\left(1-a_{n}^{\pi}\right) \mathscr{A} \cap a_{0}^{\pi} \mathscr{A}=\{0\}$ for $n$ large enough.

(2) If $a_{0}^{\pi}$ is a finite idempotent in $\mathscr{A}$ then $\lim _{n \rightarrow \infty} a_{n}^{D}=a_{0}^{D}$ if and only if $a_{n}^{\pi} \sim a_{0}^{\pi}$ for $n$ large enough.

(3) If $M(\mathscr{A}) \neq \phi$ and $\theta\left(a_{0}^{\pi} \mathscr{A}\right)<+\infty$ then $\lim _{n \rightarrow \infty} a_{n}^{D}=a_{0}^{D}$ if and only if $\theta\left(a_{n}^{\pi} \mathscr{A}\right)=\theta\left(a_{0}^{\pi} \mathscr{A}\right)$ for sufficiently large $n$.

PROOF. (1) The "if" part comes from Corollary 4.3. On the other hand, since $a_{n}^{l} \mathscr{A}=\left(1-a_{n}^{\pi}\right) \mathscr{A}$ for $n \geq 0$ and $\lim _{n \rightarrow \infty} a_{n}^{D}=a_{0}^{D}$, it follows from Lemma 2.4 that $\lim _{n \rightarrow \infty} \hat{\delta}\left(a_{n}^{l} \mathscr{A}, a_{0}^{l} \mathscr{A}\right)=0$ and consequently $a_{n}^{l} \mathscr{A} \cap\left(1-a_{0}^{l}\left(a_{0}^{l}\right)^{\#}\right) \mathscr{A}=\{0\}$ for $n$ large enough by Proposition 2.5 , that is, $\left(1-a_{n}^{\pi}\right) \mathscr{A} \cap a_{0}^{\pi} \mathscr{A}=\{0\}$.

(2) If $a_{n}^{\pi} \sim a_{0}^{\pi}$ for $n$ large enough then $a_{n}^{l}$ is a stable perturbation of $a_{0}^{l}$ by Theorem 3.3. Thus $\lim _{n \rightarrow \infty} a_{n}^{D}=a_{0}^{D}$ by Corollary 4.3.

On the other hand, if $\lim _{n \rightarrow \infty} a_{n}^{D}=a_{0}^{D}$ then for sufficiently large $n$ we have $\left\|a_{n}^{\pi}-a_{0}^{\pi}\right\|<\left\|2 a_{0}^{\pi}-1\right\|^{-1}$. Put $z_{n}=1+\left(2 a_{0}^{\pi}-1\right)\left(a_{n}^{\pi}-a_{0}^{\pi}\right)$. Then $\left\|1-z_{n}\right\|<1$, that is, $z_{n} \in \mathrm{GL}(\mathscr{A})$ and $z_{n} a_{n}^{\pi} z_{n}^{-1}=a_{0}^{\pi}$.

(3) If $\theta\left(a_{n}^{\pi} \mathscr{A}\right)=\theta\left(a_{0}^{\pi} \mathscr{A}\right)$ for $n$ large enough then $\lim _{n \rightarrow \infty} a_{n}^{D}=a_{0}^{D}$ by Theorem 3.4 and Corollary 4.3. Conversely, when $\lim _{n \rightarrow \infty} a_{n}^{D}=a_{0}^{D}$ we have $a_{n}^{\pi} \approx a_{0}^{\pi}$ for sufficiently large $n$ by the proof of (2) and hence $\theta\left(a_{n}^{\pi} \mathscr{A}\right)=\theta\left(a_{0}^{\pi} \mathscr{A}\right)$.

REMARK. Corollary 4.4 (1) covers [10, Corollary 3.4] and Corollary 4.4 (2), (3) covers [19, Theorem 2].

\section{Acknowledgements}

The author would like to thank the referee for his (or her) kindly pointing out many errors in the paper.

Part of this work was carried out while the author was visiting the Mathematical Institute, University of Munster. He wishes to express his gratitude to Professor J. Cuntz for his hospitality and his helpful suggestions.

\section{References}

[1] B. A. Barnes, 'The Fredholm elements of a ring', Canad. J. Math. 21 (1969), 84-95.

[2] N. Castro-González and J. J. Koliha, 'Perturbation of Drazin inverse for closed linear operators', Integral Equations Operator Theory 36 (2000), 92-106.

[3] N. Castro-González, J. J. Koliha and V. Rakočević, 'Continuity and general perturbation of Drazin inverse for closed linear operators', Abstr. Appl. Anal. 7 (2002), 355-347. 
[4] N. Castro-González, J. J. Koliha and Y. Wei, 'Error bounds for perturbation of the Drazin inverse of closed operators with equal spectral idempotents', Appl. Anal. 81 (2002), 915-928.

[5] G. Chen, M. Wei and Y. Xue, 'Perturbation analysis of the least squares solution in Hilbert spaces', Linear Algebra Appl. 244 (1996), 69-80.

[6] G. Chen and Y. Xue, 'Perturbation analysis for the operator equation $T x=b$ in Banach spaces', J. Math. Anal. Appl. 212 (1997), 107-125.

[7] _ - 'The expression of the generalized inverse of the perturbed operator under type I perturbation in Hilbert spaces', Linear Algebra Appl. 285 (1998), 1-6.

[8] J. Ding and L. J. Huang, 'Perturbation of generalized inverses of linear operators in Hilbert spaces', J. Math. Anal. Appl. 198 (1996), 506-515.

[9] M. P. Drazin, 'Pseudo-inverses in associative rings and semigroups', Amer. Math. Monthly 65 (1958), 506-514.

[10] V. Rakoč ević, 'Continuity of the Drazin inverse', J. Operator Theory 41 (1999), 55-68.

[11] R. Harte and M. Mbekhta, 'On generalized inverse in $C^{*}$-algebras', Studia Math. 103 (1992), 71-77.

[12] — 'On generalized inverse in $C^{*}$-algebras (II)', Studia Math. 106 (1993), 129-138.

[13] J. J. Koliha, 'Error bounds for a general perturbation of Drazin inverse', Appl. Math. Comput. 126 (2002), 181-185.

[14] J. J. Koliha and V. Rakočević, 'Continuity of the Drazin inverse II', Studia Math. 131 (1998), 167-177.

[15] G. K. Pedersen, $C^{*}$-algebras and Their Automorphism Groups (Academic Press, Boston, 1979).

[16] V. Rakočević, 'On the continuity of the Moore-Penrose inverse in $C^{*}$-algebras', Math. Montisnigri 2 (1993), 89-92.

[17] V. Rakočević and Y. Wei, 'The perturbation theory for the Drazin inverse and its applications (II)', J. Aust. Math. Soc. 70 (2001), 189-197.

[18] C. Rickart, General Theory of Banach Algebras (Van Nostrand Reinhold, New York, 1960).

[19] S. Roch and B. Silbermann, 'Continuity of generalized inverses in Banach algebras', Studia Math. 136 (1999), 197-227.

[20] Y. Xue and G. Chen, 'Some equivalent conditions of stable perturbation of operators in Hilbert spaces', Applied Math. Comput. 147 (2004), 765-772.

Department of Mathematics

East China Normal University

Shanghai 200062

China

e-mail: xyf63071@public9.sta.net.cn,yfxue@math.edu.cn. 